\title{
INFLUENCIA DE LA SUSTITUCIÓN PARCIAL DE LA HARINA DE TRIGO POR HARINA DE QUINOA Y PAPA EN LAS PROPIEDADES TERMOMECÁNICAS Y DE PANIFICACIÓN DE MASAS
}

\section{INFLUENCE OF THE PARTIAL SUBSTITUTION OF WHEAT FLOUR FOR QUINOA AND POTATO FLOUR ON THE THERMOMECHANICAL AND BREADMAKING PROPERTIES OF DOUGH}

\author{
Eduardo Rodríguez-Sandoval ${ }^{1}$, Alexandra Lascano², Galo Sandoval ${ }^{3}$
}

\begin{abstract}
1Ingeniero Químico, Doctor en Ingeniería. Docente Asociado. Departamento de Ingeniería Agrícola y Alimentos. Facultad de Ciencias Agropecuarias. Universidad Nacional de Colombia. Núcleo el Volador. Medellín, Antioquia - Colombia. Email: edrodriguezs@ unal.edu.co 2Ingeniera de Alimentos. Unidad Operativa de Investigación en Tecnología de Alimentos (UOITA). Facultad de Ciencia e Ingeniería de Alimentos. Universidad Técnica de Ambato. Ambato, Tungurahua - Ecuador. Email: alexlasca4@hotmail.com 3Ingeniero de Alimentos. Investigador de la Unidad Operativa de Investigación en Tecnología de Alimentos (UOITA). Facultad de Ciencia e Ingeniería de Alimentos. Universidad Técnica de Ambato. Ambato, Tungurahua - Ecuador. Email: sandovalgalo@yahoo.es
\end{abstract}

Rev. U.D.C.A Act \& Div. Cient. 15(1): 199 - 207, 2012

\section{RESUMEN}

Las importaciones de trigo para panificación se podrían disminuir utilizando harinas de otros recursos amiláceos, como la papa y la quinoa. En este trabajo, se evaluaron las propiedades termomecánicas de la masa y las características físicas del pan elaborado con harina compuesta de quinoatrigo y papa-trigo. Los porcentajes de sustitución aplicados fueron de 10 y $20 \%$, tanto para la harina de papa como para la de quinoa. Las propiedades funcionales de las harinas, se midieron mediante el índice de absorción de agua (IAA), índice de solubilidad de agua (ISA) y poder de hinchamiento $(\mathrm{PH})$. Las propiedades termomecánicas de las harinas compuestas, se estimaron empleando un Mixolab y las características físicas evaluadas en el producto final fueron peso, altura, ancho y volumen. Los resultados arrojaron que los mayores valores de IAA $(4,48)$, ISA $(7,45 \%)$ y $\mathrm{PH}$ $(4,84)$ fueron para la harina de papa. La harina compuesta con quinoa presentó los menores valores de asentamiento y estabilidad de la cocción, lo cual, es un buen indicador de conservación del pan. Por el contrario, la harina compuesta de papa-trigo mostró los menores valores de estabilidad, torque mínimo y torque de pico y el mayor porcentaje de absorción de agua. La harina compuesta de papa-trigo, con un grado de sustitución de $10 \%$, presentó los resultados de peso $(133,86 \mathrm{~g})$, ancho $(6,01 \mathrm{~cm})$ y volumen $\left(491,67 \mathrm{~g} / \mathrm{cm}^{3}\right)$, más parecidos a la muestra control de harina de trigo.
Palabras clave: Mixolab, reología, harina compuesta, almidón.

\section{SUMMARY}

The imports of wheat for breadmaking could be reduced by using flour from other starch sources such as potato and quinoa.The thermomechanical properties of dough and the physical characteristics of bread, made from quinoawheat and potato-wheat composite flours were evaluated in this study. The percentages of substitution used were 10 and $20 \%$ for both potato and quinoa flour. The functional properties of flours were measured by the water absorption index (IAA), water solubility index (ISA) and swelling power $(\mathrm{PH})$. The thermomechanical properties of composite flours were assessed using a Mixolab and the baking quality characteristics evaluated of the final product were weight, height, width and volume. The results showed that the higher values of IAA (4.48), ISA (7.45\%) and PH (4.84) were for potato flour. Quinoa-wheat composite flour presented the lower setback and cooking stability data, which is a good indicative of shelf life of bread. On the other hand, potatowheat composite flour showed the lower stability, minimum torque and peak torque, and the higher water absorption. Potato-wheat composite flour substituted for $10 \%$, presented the weight $(133.86 \mathrm{~g})$, width $(6.01 \mathrm{~cm})$ and volume $(491.67 \mathrm{~g} /$ $\mathrm{cm}^{3}$ ) results more similar to wheat bread. 
Key words: Mixolab, rheology, composite flour, starch.

\section{INTRODUCCIÓN}

En la mayoría de países Andinos existe un amplio desbalance entre la producción y la demanda del grano para abastecer las necesidades internas de harina de trigo, utilizada en panificación. Las principales causas son la falta de tierras adecuadas para el cultivo del cereal, los bajos rendimientos, los precios internacionales fluctuantes, los subsidios y la tecnología de los países desarrollados (Henao-Osorio \& Aristizábal-Galvis, 2009). Una alternativa para reemplazar parte de estas importaciones sería sustituir la harina de trigo, utilizada en productos de panificación, por harinas provenientes de otros recursos amiláceos, como cereales diferentes, raíces, tubérculos y leguminosas; sin embargo, las propiedades específicas de la harina de trigo la hacen indispensable en la preparación de productos aireados, tales como el pan, por la habilidad de sus proteínas (gluten) para formar una red continua, que se puede extender y retener burbujas de gas, producidas durante la fermentación (Scanlon \& Zghal, 2001).

La quinoa (Chenopodium quinoa Willd.) es un pseudocereal originario de Suramérica y se cultiva, principalmente, en Perú, en Bolivia, en Ecuador y en el sur de Colombia. Existe un especial interés en este pseudocereal, debido a su alto contenido en proteína y minerales (Park \& Morita, 2005). Su composición proximal varía con el cultivar, pero, en general, presenta de 10 a $18 \%$ de proteína, de 4 a $8 \%$, de grasa, de 54 a $64 \%$ de carbohidratos, de 2 a $4 \%$ de cenizas y de 2 a $5 \%$ de fibra cruda. Su balance de aminoácidos es mejor que en el trigo y el maíz, porque la lisina, principal aminoácido limitante, se muestra en cantidades considerables (Lorenz \& Coulter, 1991). Prevalecen diferentes investigaciones, en las cuales, se ha empleado la harina de quinoa para la producción de pan, teniendo muy buenos resultados, con sustituciones menores al 10\% (Lorenz \& Coulter, 1991; Chauhan et al. 1992; Morita et al. 2001; Park et al. 2005; Rosell et al. 2009).

Por otro lado, la papa (Solanum tuberosum) es uno de los productos básicos más cultivados y consumidos del planeta, después del trigo, el arroz y el maíz (Navarre et al. 2011). La papa contiene una buena calidad de proteína, de fibra dietaria, de minerales y de poca o despreciable cantidad de grasa (Misra \& Kulshrestha, 2003). La harina de papa, se ha convertido en el producto con valor agregado más difundido, debido a su versatilidad, ya que funciona como mejorador de sabor, del color y como espesante; además, se ha manipulado en salsas, en productos de panadería, en extruidos, en pasabocas y en sopas instantáneas, entre otros (Yadav et al. 2006). En el proceso de elaboración de pan, la papa se puede adicionar a la harina de trigo como almidón y/o harina nativa o precocida. Los resultados más adecuados en la sustitución con papa, se han encontrado por debajo de $20 \%$, puesto que en niveles superiores producen panes con características inaceptables, en términos de volumen, de textura y de sabor (Cerón et al. 2011; Greene \& Bovell-Benjamin, 2004). Kaack et al. (2006) concluyeron que una fibra de papa solubilizada y secada por aspersión, se puede utilizar para sustituir un $12 \%$ de harina de trigo en la elaboración de pan, con buenas características organolépticas.

Las propiedades reológicas de la masa pueden predecir el comportamiento del producto en panificación si el esfuerzo aplicado y la deformación están en el mismo rango de las utilizadas en el procesamiento real. Además, las características de empastamiento, se correlacionan con el envejecimiento del pan (Collar, 2003). Uno de los equipos que se ha empleado, últimamente, para evaluar las materias primas utilizadas en panificación es el Mixolab, el cual, permite caracterizar el comportamiento fisicoquímico y reológico de las masas cuando se someten simultáneamente a mezclado y a temperaturas programadas. Por consiguiente, es posible registrar los cambios mecánicos, debido al mezclado y al calentamiento, así como el trabajo mecánico y las condiciones de calor que se podrían esperar durante el proceso de horneo (Rosell et al. 2007). Teniendo en cuenta lo expuesto, se propone en este trabajo evaluar el perfil termomecánico de las harinas compuestas y las características del pan elaborado con una sustitución del 10 y $20 \%$ de harina de quinoa y de papa.

\section{MATERIALES Y MÉTODOS}

Materia prima: Se utilizó harina de trigo fortificada con calcio, con tiamina, con riboflavina, con niacina, con hierro y con ácido fólico, proveniente de Molinos del Ecuador C.A. (Guayaquil, Ecuador) y papa de la variedad Gabriela, cultivada en Quero, provincia de Tungurahua, Ecuador (altura > a 3000msnm). La papa fue seleccionada y lavada con agua potable; posteriormente, se le retiró la cáscara con un pelador de papa abrasivo (Proingal, Quito - Ecuador), se dejó en una solución de $\mathrm{NaCl}(4 \% \mathrm{p} / \mathrm{p})$, por 5 min y se cortó en bastones de 3 a $5 \mathrm{~cm}$ de largo y $1 \mathrm{~cm}$ de ancho, que se agregaron a una solución de eritorbato de sodio $(0,4 \% \mathrm{p} / \mathrm{p})$, por 15 min. La precocción de los trozos de papa, se realizó en una autoclave a $105^{\circ} \mathrm{C}$, durante $6 \mathrm{~min}$. Luego, las muestras se secaron a $45^{\circ} \mathrm{C}$, hasta un contenido de humedad de 10 a 13\%. Los bastones secos se molieron en un molino de discos (Proingal, Quito, Ecuador). La harina obtenida, se clasificó en un tamizador (Proingal, Quito, Ecuador) hasta obtener un tamaño de partícula menor a $180 \mathrm{~mm}$. La harina de papa, se almacenó en bolsas de polietileno de alta densidad (Sandoval, 2007).

La quinoa, variedad Tuncahuán, fue adquirida en Colta, provincia de Chimborazo, Ecuador, cultivada a 2800msnm. Los granos se seleccionaron, se limpiaron y se acondicionaron para mejorar la separación del endospermo 
del pericarpio. El acondicionamiento consistió en remojar los granos hasta que alcanzaran una humedad del $16 \%$. El grano, se molió al pasar por cilindros de rotura, con un ángulo de inclinación específico, para mantener un nivel equilibrado entre extracción y cenizas. En la molienda, se controló el sobrecalentamiento, para prevenir daños en la proteína y evitar un exceso de deshidratación. La harina integral, se clasificó en un tamizador (Proingal, Quito, Ecuador), hasta obtener un tamaño de partícula menor a $180 \mathrm{~mm}$. Las dos harinas, se mezclaron con la harina de trigo, en proporciones de 10 y $20 \%$ (p/p), tomando como criterio ensayos preliminares de panificación y de estudios realizados previamente (Cerón et al. 2011; Park \& Morita, 2005).

Propiedades fisicoquímicas: El contenido de humedad de la harina de trigo, de la papa y de la quinoa, se determinó en un analizador de humedad (MLS50-3, Kern \& Son $\mathrm{GmbH}$, Balingen, Alemania). El resultado fue el promedio de tres repeticiones. Las pruebas de índice de absorción de agua (IAA), índice de solubilidad de agua (ISA) y poder de hinchamiento $(\mathrm{PH})$, se pueden utilizar como un indicativo del grado de modificación de los almidones por tratamientos termomecánicos. El método que se siguió para la determinación de IAA, ISA y PH fue tomado de Anderson et al. (1969), con algunas modificaciones. La harina, se pasó por una malla de $180 \mathrm{~mm}$, para normalizar el tamaño de la muestra. $0,5 \mathrm{~g}$ (b.s.) de muestra, se pesaron en tubos de centrífuga, utilizando una balanza analítica (BBL31 Boeco, Beockel+Co, Alemania). Se adicionó 6ml de agua destilada a $30^{\circ} \mathrm{C}$ y se incubó en el baño con agitación (WiseBath, Wisd Laboratory Instruments, USA), durante 30 min. Posteriormente, se centrifugó a 5000 rpm, por 20 min, en una centrífuga (EBA 12, Hettich Zentrifugen, Tuttlingen, Alemania). El sobrenadante se decantó, se midió su volumen, se filtró y se tomó $2 \mathrm{ml}$ de filtrado para secarlo a $90^{\circ} \mathrm{C}$, por 4 h. El gel retenido en los tubos se pesó. El IAA, ISA y PH, se determinaron de acuerdo a las ecuaciones No.1, 2 y 3. Los ensayos se realizaron por triplicado.

$$
I A A=\frac{\text { Peso.gel }(g)}{\text { Peso.muestra }(g)}
$$

$\operatorname{ISA}(\%)=\frac{\text { Peso.sec } \text { o sobrenadane }(g)}{\text { Peso.muestra }(g)} * 100$

$$
P H=\frac{\text { Peso.gel }(g)}{\text { Peso.muestra }(g)-\text { Peso.seco.sobrenadane }(g)}
$$

Determinación de las propiedades termomecánicas utilizando el Mixolab: El comportamiento del mezclado y el empastamiento de las mezclas de harinas y la harina de trigo, se evaluaron en un Mixolab (Chopin Technologies, Villeneuve la Garenne, Francia), el cual, mide en tiempo real el torque (N.m) producido por el paso de la masa entre dos cuchillas. El protocolo utilizado permite mezclar la masa bajo temperatura controlada y realizar un barrido de temperatura hasta $90^{\circ} \mathrm{C}$, seguido por un enfriamiento. Para los ensayos, se pesó una muestra de acuerdo a su contenido de humedad y el equipo hidrató dicha muestra, hasta alcanzar un torque de 1,1 N.m (Rosell et al. 2007).

Los parámetros obtenidos de la curva registrada fueron: Porcentaje de absorción de agua (\%) o porcentaje de agua requerida, para que la masa produzca un torque de 1,1 N.m; tiempo de desarrollo de la masa (min) o tiempo, para alcanzar el máximo torque a $30^{\circ} \mathrm{C}$; consistencia máxima inicial (N.m) (C1) o máximo torque que se alcanza durante el mezclado, a $30^{\circ} \mathrm{C}$; estabilidad (min) o tiempo en que el torque se mantiene en 1,1 N.m; debilitamiento mecánico (N.m) o diferencia de torque entre el máximo torque a $30^{\circ} \mathrm{C}$ y el torque después de mantenerse a $30^{\circ} \mathrm{C}$; el torque mínimo (N.m) (C2) o el valor mínimo de torque producido por la masa, cuando se somete a un esfuerzo mecánico y a un tratamiento térmico; debilitamiento térmico (N.m) o la diferencia entre el torque al final del sostenimiento a $30^{\circ} \mathrm{C}$ y el torque mínimo; torque de pico (N.m) (C3) o el torque máximo producido durante la etapa de calentamiento; estabilidad en la cocción, calculada como la razón del torque después del sostenimiento a $90^{\circ} \mathrm{C}$ y el máximo toque durante el periodo de calentamiento (C3) y, finalmente, el asentamiento (N.m) o la diferencia entre el torque producido después del enfriamiento a $50^{\circ} \mathrm{C}(\mathrm{C} 5)$ y el torque del final del periodo de calentamiento.

Pruebas de panificación: Las pruebas de panificación, se realizaron de acuerdo a la Norma Técnica Ecuatoriana (NTE) INEN 530 (1980), que consistió en mezclar 500g de harina con $100 \mathrm{~mL}$ de una solución de levadura fresca $(15 \mathrm{~g})$ y azúcar $(15 \mathrm{~g})$ a $28 \pm 5^{\circ} \mathrm{C}$. Además, se adicionó $100 \mathrm{~mL}$ de una solución de $\mathrm{NaCl}(10 \%$ p/p) y una cantidad de agua necesaria para alcanzar una masa de consistencia adecuada, según los porcentajes de absorción obtenidos en el Mixolab. El proceso de mezclado y de amasado, se realizaron durante 8,5 min, en una amasadora horizontal (Proingal, Quito, Ecuador), teniendo en cuenta en agregar $10 \mathrm{~g}$ de grasa, 2 min antes de terminar la operación. La masa obtenida, se redondeó manualmente y se situó en una cámara de fermentación (Proingal, Quito, Ecuador), a $30^{\circ} \mathrm{C}$, con una humedad de $85 \%$, durante $100 \mathrm{~min}$. Después, la muestra se amasó nuevamente a mano por 2 min, se redondeó y se colocó en la misma cámara de fermentación, por 25 min. Posteriormente, la masa se desgasificó y se dividió en cinco porciones del mismo peso. Cada una de las porciones se aplanaron con un rodillo hasta formar una lámina gruesa $(0,5$ a $1 \mathrm{~cm}$ de espesor), la cual, se enrolló y se situó en moldes, con las siguientes dimensiones: base $90 \mathrm{~mm} * 51 \mathrm{~mm}$, alto $83 \mathrm{~mm}$, superior $110 \mathrm{~mm} * 70 \mathrm{~mm}$. Las muestras, se llevaron a la cámara de fermentación durante 60 min y, luego, se horneron en un horno rotatorio (Equipan, Quito, Ecuador), a $210 \pm 5^{\circ} \mathrm{C}$, por $25 \mathrm{~min}$. El pan, se retiró del molde a los 5 min y se esperó $1 \mathrm{~h}$ para proceder a medir peso, ancho, alto y volumen aparente por desplazamiento de semillas de quinoa, en cada una de las muestras. 
Análisis estadístico: Se aplicó un diseño factorial completamente aleatorizado con dos factores: Tipo de harina (trigo, trigo-papa, trigo-quinoa) y porcentaje de sustitución (10 y 20\%). Los datos experimentales, se sometieron a ANOVA, con un nivel de significancia del $5 \%$ y se utilizó diferencia mínima significativa (LSD) para comparar los tratamientos, con diferencias significativas. El análisis estadístico, se realizó mediante Statgraphics plus 5,1. Los resultados se presentan como el promedio \pm desviación estándar.

\section{RESULTADOS Y DISCUSIÓN}

Caracterización de harinas: En la tabla 1, se reportan los valores promedios del contenido de humedad, de índice de absorción de agua (IAA) y de solubilidad en agua (ISA) y el poder de hinchamiento $(\mathrm{PH})$ de las harinas de trigo, de papa y de quinoa. Los parámetros evaluados, se afectaron significativamente por el tipo de harina $(P<0,05)$. La harina de trigo presenta los menores valores de IAA, ISA y $\mathrm{PH}$, mientras que la de papa muestra los valores más altos. El contenido de humedad es similar para las muestras de trigo y quinoa, mientras que es un poco menor para la harina de papa. Los valores de IAA para la harina de quinoa son menores a los reportados por Abugoch et al. (2009), que se encuentran entre 2,3 y 4,5; sin embargo, ellos evaluaron la capacidad de retención de agua, con un método diferente.

La gelatinización causa un incremento en el poder de hinchamiento; la solubilidad y la absorción de agua son parámetros que muestran la magnitud de la interacción entre las cadenas de almidón dentro de las secciones amorfas y cristalinas. Estas interacciones se afectan por la relación amilosa/amilopectina y por las características de la amilosa y la amilopectina, en términos del peso/distribución, grado y longitud de ramificación y conformación. El mayor poder de hinchamiento, de solubilidad y de absorción del almidón de papa, se debe, probablemente, al alto contenido de grupos fosfato en la amilopectina, los cuales, generan una repulsión en las cadenas adyacentes, incrementando la hidratación por el debilitamiento de los enlaces dentro de la parte cristalina del gránulo (Hoover, 2001). La morfología, el procesamiento y la composición de las harinas afectan sus propiedades funcionales, por consiguiente, aunque los niveles de lípidos presentes en la harina de trigo son bajos, afectan el poder de hinchamiento y la absorción de agua de este almidón (Singh et al. 2003).

Propiedades termomecánicas: Durante el mezclado inicial, la distribución del material, el rompimiento de las partículas proteicas esféricas y la hidratación de los componentes de la harina, se llevan a cabo en asocio con el reforzamiento y la alineación de las proteínas, conduciendo la formación de una estructura viscoelástica tridimensional, capaz de retener gas (Rosell et al. 2007; Collar et al. 2007; Angioloni \& Collar, 2009). La tabla 2 muestra los parámetros reológicos en la etapa de mezcla del Mixolab de la harina de trigo patrón y las harinas compuestas de trigo-papa y trigo-quinoa. No se encontró diferencias significativas en la consistencia máxima inicial (C1) y el debilitamiento mecánico $(P<0,05)$. El mayor porcentaje de absorción (\%) fue para la harina compuesta trigo-papa, con $20 \%$ de sustitución, mientras que el menor fue para trigo-quinoa, con $10 \%$. Este resultado está de acuerdo con los datos de IAA y PH, demostrando que la harina de papa afecta significativamente la absorción de la harina compuesta, debido a la relación de amilosa/amilopectina, contenido de fosforo, características moleculares de la amilosa y la amilopectina en términos de peso/distribución, grado de ramificación y conformación del almidón (Kaur et al. 2007). Los resultados del porcentaje de absorción para la harina de trigo fueron similares a los reportados en la literatura (Codină, 2008; Sun et al. 2010).

El tiempo de desarrollo fue significativamente menor para la harina compuesta trigo-papa y mayor para trigo-quinoa, con $20 \%$ de sustitución. La harina de quinoa incrementa el tiempo necesario para una completa hidratación del material, que puede estar relacionado con la composición

Tabla 1. Contenido de humedad, índice de absorción de agua (IAA), índice de solubilidad en agua (ISA) y poder de hinchamiento $(\mathrm{PH})$ de materias primas farináceas*.

\begin{tabular}{|l|c|c|c|c|}
\hline Harina & Humedad (\%) & IAA & ISA (\%) & PH \\
\hline Trigo & $12,57 \pm 0,28^{\mathrm{b}}$ & $1,92 \pm 0,06^{\mathrm{a}}$ & $2,09 \pm 0,26^{\mathrm{a}}$ & $1,96 \pm 0,07^{\mathrm{a}}$ \\
\hline Papa & $12,03 \pm 0,19 \mathrm{a}$ & $4,48 \pm 0,11^{\mathrm{c}}$ & $7,45 \pm 0,72^{\mathrm{c}}$ & $4,84 \pm 0,12^{\mathrm{c}}$ \\
\hline Quinoa & $12,47 \pm 0,12^{\mathrm{b}}$ & $2,31 \pm 0,08^{\mathrm{b}}$ & $5,10 \pm 0,12^{\mathrm{b}}$ & $2,43 \pm 0,08^{\mathrm{b}}$ \\
\hline
\end{tabular}

*Las letras no comunes indican diferencia significativa, según la LSD de Fisher al 95\%. 
Tabla 2. Parámetros reológicos del área de mezcla del Mixolab en la harina de trigo y las harinas compuestas de trigo-papa y trigo-quinoa*.

\begin{tabular}{|l|l|l|l|l|l|l|}
\hline Harina & $\begin{array}{l}\text { Sustitución } \\
(\%)\end{array}$ & $\begin{array}{l}\text { Absorción de } \\
\text { agua (\%) }\end{array}$ & $\begin{array}{l}\text { Tiempo de } \\
\text { desarrollo } \\
(\mathrm{min})\end{array}$ & $\begin{array}{l}\text { Consistencia } \\
\text { máxima inicial } \\
(\mathrm{C} 1)(\text { N.m) }\end{array}$ & $\begin{array}{l}\text { Estabilidad } \\
(\mathrm{min})\end{array}$ & $\begin{array}{l}\text { Debilitamiento } \\
\text { mecánico (N.m) }\end{array}$ \\
\hline Trigo & 0 & $61,93 \pm 0,1^{\mathrm{b}}$ & $6,21 \pm 0,09^{\mathrm{b}}$ & $1,12 \pm 0,03^{\mathrm{a}}$ & $8,58 \pm 0,41^{\mathrm{b}}$ & $0,08 \pm 0,01^{\mathrm{a}}$ \\
\hline \multirow{2}{*}{ Papa } & 10 & $64,5 \pm 0,02^{\mathrm{c}}$ & $1,24 \pm 0,13^{\mathrm{a}}$ & $1,09 \pm 0,03^{\mathrm{a}}$ & $8,95 \pm 0,04^{\mathrm{b}}$ & $0,05 \pm 0,06^{\mathrm{a}}$ \\
\cline { 2 - 7 } & 20 & $68,7 \pm 0,01^{\mathrm{d}}$ & $0,98 \pm 0,01^{\mathrm{a}}$ & $1,08 \pm 0,01^{\mathrm{a}}$ & $7,3 \pm 0,21^{\mathrm{a}}$ & $0,03 \pm 0,01^{\mathrm{a}}$ \\
\hline \multirow{2}{*}{ Quinoa } & 10 & $60,4 \pm 0,42^{\mathrm{a}}$ & $6,65 \pm 0,14^{\mathrm{bc}}$ & $1,11 \pm 0,02^{\mathrm{a}}$ & $10,26 \pm 0,25^{\mathrm{c}}$ & $0,02 \pm 0,02^{\mathrm{a}}$ \\
\cline { 2 - 7 } & 20 & $61,0 \pm 0,01^{\mathrm{ab}}$ & $7,1 \pm 0,71^{\mathrm{c}}$ & $1,11 \pm 0,01^{\mathrm{a}}$ & $10,22 \pm 0,02^{\mathrm{c}}$ & $0,05 \pm 0,01^{\mathrm{a}}$ \\
\hline
\end{tabular}

*Las letras no comunes indican diferencia significativa, según la LSD de Fisher al 95\%.

y las características del almidón de este pseudocereal. El almidón de quinoa tiene una baja solubilidad y bajo poder de hinchamiento, debido a las fuertes fuerzas de unión o los entrecruzamientos dentro del gránulo de almidón (Ruales \& Nair, 1994; Ahamed et al. 1996).

La menor estabilidad fue para trigo-papa, con $20 \%$ y la mayor fue para trigo-quinoa. El bajo tiempo de estabilidad durante el periodo de mezcla es un indicativo de la debilidad estructural de la red de gluten en la masa (Park \& Morita, 2005). La adición de harina de quinoa incrementa significativamente la estabilidad; sin embargo, Morita et al. (2001) reportaron que la estabilidad de la masa en el farinógrafo decrece a medida que se aumenta la sustitución de la harina de quinoa, de 7,5 a $20 \%$. La estabilidad del trigo patrón fue similar a los resultados reportados en investigaciones previas con otras clases de trigo (Angioloni \& Collar, 2009; Sun et al. 2010). Por otro lado, cuando el mezclado es excesivo, la fuerza de la masa decrece, principalmente, por el debilitamiento de la proteína, debido al esfuerzo de corte mecánico. Los valores del debilitamiento mecánico de la harina de trigo patrón, como de las harinas compuestas, son muy bajos en comparación a los reportados por Rosell et al. (2007), que están entre 0,28 a 0,33 N.m.

La tabla 3 presenta los parámetros reológicas del área de empastamiento del Mixolab en la harina de trigo patrón y las harinas compuestas de trigo-papa y trigo-quinoa. Los parámetros de torque mínimo (C2) y debilitamiento térmico están muy relacionados, afectándose significativamente por el tipo de harina sustituida (quinoa y papa), pero no por el porcentaje de sustitución. El torque mínimo (C2) es menor para harina compuesta de trigo-papa, mientras que es similar para la harina de trigo y harina compuesta de trigoquinoa. Estos valores son similares a resultados obtenidos en la literatura, encontrándose entre 0,26 a 0,47 N.m (Rosell et al. 2007; Angioloni \& Collar, 2009; Sun et al. 2010). Por otro lado, el debilitamiento térmico es menor para trigo seguido de la harina compuesta trigo-quinoa, mientras que el mayor valor lo registró la mezcla trigo-papa. Estos valores son mayores a los reportados por Rosell et al. (2007) para harinas de trigo con diferentes hidrocoloides, los cuales, se ubican entre 0,24 a 0,4 N.m. El efecto combinado del esfuerzo de corte mecánico y la temperatura impuesta en el equipo producen una reducción en el torque, que se podría relacionar con el comienzo de la desestabilización y el desdoblamiento de la proteína (Rosell et al. 2007; Angioloni \& Collar, 2009).

El tipo de harina adicionada y el porcentaje sustituido afectaron significativamente los resultados de torque de pico (C3). La harina compuesta con quinoa tuvo mayores valores con respecto a la adicionada con papa; además, un porcentaje de $10 \%$ de sustitución presentó los mayores resultados de C3. Los valores obtenidos en este trabajo son similares a los encontrados en otros estudios, que se encuentran entre 1,4-1,9 N.m (Rosell et al. 2007; Rosell et al. 2009; Angioloni \& Collar, 2009). La harina compuesta con quinoa al $10 \%$ también presentó los valores más cercanos a la de trigo, siendo similar a los resultados reportados en la literatura (Park \& Morita, 2005; Rosell et al. 2009). A medida que el porcentaje de sustitución aumentó, el valor de C3 disminuyó, contrario al comportamiento mostrado por Rosell et al. (2009). Esta diferencia puede ser debida a la diferencia en el cultivar utilizado o al proceso de obtención de la harina de quinoa, en la cual, se aplicó un acondicionamiento con agua a $50^{\circ} \mathrm{C}$, durante $4 \mathrm{~h}$. Por otro lado, a medida que se aumentó la sustitución de harina de papa, el valor de C3 disminuyó, contrario a lo reportado por Zaidul et al. (2007), 
Tabla 3. Parámetros reológicos del área de empastamiento del Mixolab en la harina de trigo, y las harinas compuestas de trigo-papa y trigo-quinoa*.

\begin{tabular}{|l|l|l|l|l|l|l|}
\hline Harina & $\begin{array}{l}\text { Sustitución } \\
(\%)\end{array}$ & $\begin{array}{l}\text { T o r q u e } \\
\text { mínimo (C2) } \\
(\text { N.m) }\end{array}$ & $\begin{array}{l}\text { Debilitamiento } \\
\text { térmico (N.m) }\end{array}$ & $\begin{array}{l}\text { Torque de } \\
\text { pico (C3) } \\
\text { (N.m) }\end{array}$ & $\begin{array}{l}\text { Estabilidad en } \\
\text { la cocción }\end{array}$ & $\begin{array}{l}\text { Asentamiento } \\
\text { (N.m) }\end{array}$ \\
\hline Trigo & 0 & $0,45 \pm 0,02^{\mathrm{b}}$ & $0,59 \pm 0,01^{\mathrm{a}}$ & $1,84 \pm 0,04^{\mathrm{d}}$ & $0,98 \pm 0,01^{\mathrm{c}}$ & $0,76 \pm 0,05^{\mathrm{d}}$ \\
\hline \multirow{2}{*}{ Papa } & 10 & $0,37 \pm 0,01^{\mathrm{a}}$ & $0,67 \pm 0,03^{\mathrm{c}}$ & $1,51 \pm 0,01^{\mathrm{b}}$ & $0,98 \pm 0,01^{\mathrm{c}}$ & $0,43 \pm 0,02^{\mathrm{b}}$ \\
\cline { 2 - 7 } & 20 & $0,34 \pm 0,01^{\mathrm{a}}$ & $0,71 \pm 0,02^{\mathrm{c}}$ & $1,18 \pm 0,01^{\mathrm{a}}$ & $0,99 \pm 0,02^{\mathrm{c}}$ & $0,40 \pm 0,02^{\mathrm{b}}$ \\
\hline \multirow{2}{*}{ Quinoa } & 10 & $0,45 \pm 0,02^{\mathrm{b}}$ & $0,64 \pm 0,02^{\mathrm{b}}$ & $1,80 \pm 0,04^{\mathrm{d}}$ & $0,93 \pm 0,01^{\mathrm{b}}$ & $0,47 \pm 0,01^{\mathrm{c}}$ \\
\cline { 2 - 7 } & 20 & $0,45 \pm 0,01^{\mathrm{b}}$ & $0,62 \pm 0,01^{\mathrm{b}}$ & $1,69 \pm 0,01^{\mathrm{c}}$ & $0,89 \pm 0,01^{\mathrm{ab}}$ & $0,30 \pm 0,01^{\mathrm{a}}$ \\
\hline
\end{tabular}

*Las letras no comunes indican diferencia significativa, según la LSD de Fisher al 95\%.

quienes analizaron el perfil de empastamiento de una mezcla de harina de trigo y almidón de papa. Esta diferencia, se debe a que la harina de papa utilizada en este estudio tuvo un proceso de precocción, afectando las características funcionales del almidón.

A medida que la temperatura se incrementa, el papel de las proteínas queda en un segundo plano, siendo la gelatinización del almidón el principal responsable, por las variaciones del torque. Durante esta etapa, los gránulos de almidón absorben el agua disponible en el medio y se hinchan, mientras que las cadenas de amilosa se lixivian en la fase acuosa intergranular, promoviendo el incremento en la viscosidad y, por consiguiente, el aumento en el torque. Esto sucede hasta que el esfuerzo de corte mecánico y la temperatura programada en el equipo producen el rompimiento físico de los gránulos, los cuales, se asocian con una reducción en la viscosidad (Rosell et al. 2007).

En una curva de empastamiento de almidón, la estabilidad en la cocción se relaciona con la estabilidad de los gránulos de almidón quebrados en las temperaturas de calentamiento programadas (Rojas et al. 1999). La estabilidad en la cocción fue similar para la harina de trigo y la harina compuesta de trigo-papa y fue menor para la harina compuesta de quinoa. Altas viscosidades durante el empastamiento y las bajas viscosidades después del periodo de sostenimiento a $90^{\circ} \mathrm{C}$ de las suspensiones de trigo, se consideran como los parámetros que predicen el comportamiento de firmeza del pan durante el almacenamiento (Collar, 2003). Bajas estabilidades de cocción se relacionan con un aumento en el tiempo de vida útil de un pan, por lo tanto, se podría afirmar que los panes sustituidos con quinoa conservarían, por mayor tiempo, las características de firmeza del producto, durante el almacenamiento.
El asentamiento fue menor para la mezcla de trigo-quinoa y mayor para la harina de trigo. La harina de quinoa tuvo una baja consistencia durante el enfriamiento, sobre todo, con $20 \%$ de sustitución, que indica una baja recristalización o retrogradación. Estos resultados fueron similares a los encontrados por Rosell et al. (2009). Por otro lado, la harina compuesta con papa presentó menores valores de asentamiento que la de trigo, siendo contrario a los resultados reportados por Zaidul et al. (2007), quienes hallaron un asentamiento mayor para la mezcla de harina de trigo-almidón de papa, además, aumentaba a medida que se incrementaba la sustitución de papa de 10 a 30\%. Esta diferencia puede ser debido a que la harina de papa utilizada en este estudio es precocida, afectando sus características de empastamiento y generando menores efectos en el enfriamiento, por la falta de liberación de amilosa. Los parámetros aconsejables para retardar el envejecimiento del pan y obtener alta puntuación sensorial relacionada con la miga y la corteza, constan de alta temperatura de empastamiento, alta viscosidad durante el empastamiento y gelación y baja viscosidad después del periodo de calentamiento (Collar, 2003).

Parámetros de panificación: Las pruebas de panificación evaluadas, se presentan en la tabla 4 . No hay diferencia significativa en el ancho de los panes elaborados con las diferentes harinas. Por otro lado, los productos a partir de harina compuesta de trigo-quinoa, con un $10 \%$ de sustitución, tuvieron la mayor altura, seguida de la harina compuesta de trigo-papa, al $10 \%$. Los panes de trigo y trigo-quinoa, con $20 \%$ de sustitución, tienen una altura similar. Al aumentar el porcentaje de sustitución, aumenta significativamente el peso de las muestras, sobre todo cuando se adiciona harina de papa, al 20\%. El menor peso obtenido fue para los panes a partir de trigo-quinoa, con $10 \%$, mientras que el mayor fue para las muestras de harina compuesta trigo-papa, con $20 \%$ 
Tabla 4. Parámetros de panificación de panes elaborados a partir de harina de trigo y harinas compuestas de trigo-papa y trigo-quinoa*.

\begin{tabular}{|l|c|l|l|l|l|}
\hline Harina & \% Sustitución & Peso $(\mathrm{g})$ & Ancho $(\mathrm{cm})$ & Alto $(\mathrm{cm})$ & Volumen $\left(\mathrm{cm}^{3}\right)$ \\
\hline Trigo & 0 & $132,86 \pm 0,88^{\mathrm{b}}$ & $6,02 \pm 0,28^{\mathrm{a}}$ & $8,97 \pm 0,63^{\mathrm{b}}$ & $485,0 \pm 26,4^{\mathrm{d}}$ \\
\hline \multirow{2}{*}{ Papa } & 10 & $133,86 \pm 1,13^{\mathrm{b}}$ & $6,01 \pm 0,17^{\mathrm{a}}$ & $9,57 \pm 0,49 \mathrm{c}$ & $491,67 \pm 14,3^{\mathrm{d}}$ \\
\cline { 2 - 6 } & 20 & $140,61 \pm 1,97^{\mathrm{c}}$ & $5,95 \pm 0,18^{\mathrm{a}}$ & $7,84 \pm 0,37^{\mathrm{a}}$ & $461,25 \pm 19,8^{\mathrm{c}}$ \\
\hline \multirow{2}{*}{ Quinoa } & 10 & $130,80 \pm 1,26^{\mathrm{a}}$ & $6,20 \pm 0,32^{\mathrm{a}}$ & $9,94 \pm 0,68^{\mathrm{d}}$ & $434,44 \pm 19,3^{\mathrm{b}}$ \\
\cline { 2 - 6 } & 20 & $133,65 \pm 1,73^{\mathrm{b}}$ & $6,03 \pm 0,18^{\mathrm{a}}$ & $8,89 \pm 0,4^{\mathrm{b}}$ & $362,5 \pm 15,1^{\mathrm{a}}$ \\
\hline
\end{tabular}

*Las letras no comunes indican diferencia significativa, según la LSD de Fisher al 95\%.

y los demás presentaron pesos similares. El menor peso y la mayor altura para las muestras sustituidas con harina de quinoa al $10 \%$ son evidencia de espacios de aire visibles en la miga, lo cual, puede ser generado por el aumento de la cantidad de azúcares fermentables a partir del almidón, debido a la alta actividad de alfa amilasas en la harina de quinoa (Lorenz \& Coulter, 1991).

El volumen de la miga es una de las características más importantes de un pan, porque ofrece una medida cuantitativa del proceso de panificación. Además, este parámetro es muy importante para los consumidores, debido a que se relaciona con la percepción de un producto ligero, pero no denso, es decir, características de densidad y de volumen de la miga se asocian con un producto de panadería específico (Hathorn et al. 2008). Al comparar el volumen de las muestras, el producto a partir de harina compuesta de trigo-quinoa tuvo los menores valores, mientras que los mayores volúmenes fueron para los panes de harina de trigo y harina compuesta de trigo-papa, a 10\%. Se debe resaltar que la desviación estándar de este parámetro es alta si se compara con las demás características evaluadas en el pan, mostrando amplias diferencias que existen aun en un mismo tratamiento.

Los resultados del pan con harina de quinoa están de acuerdo a las investigaciones realizadas previamente (Lorenz \& Coulter, 1991; Chauhan et al. 1992; Morita et al. 2001; Park et al. 2005; Rosell et al. 2009), donde al aumentar el porcentaje de sustitución por encima del $10 \%$ disminuye el volumen de la miga, debido a que la harina de quinoa no tiene proteína formadora de gluten, como la harina de trigo; además, se presenta un aumento en la proteína insoluble alcalina, que se correlaciona estrechamente con una pobre calidad de mezclado de la masa (Park et al. 2005). Morita et al. (2001) demostró que una masa de trigo, con $20 \%$ de harina de quinoa, presenta una estructura irregular, con capas de gluten, sin una distribución continua y rodeadas, con amplios gránulos de almidón.

El mayor volumen obtenido fue para la muestra con un $10 \%$ de harina de papa. Este resultado, se podría deber a que las enzimas amilolíticas presentes en la papa rompen la estructura del gránulo de almidón, produciendo azúcares simples y sustratos fermentables para la levadura y, así, incrementa la velocidad de fermentación (Greene \& BovellBenjamin, 2004); sin embargo, al aumentar la concentración de harina de papa por encima de $10 \%$ disminuye el volumen de la muestra, debido al bajo contenido de gluten.

El porcentaje de sustitución de harina de trigo (10 y $20 \%$ ) con harinas de quinoa y papa producen masas con diferentes perfiles termomecánicos y panes con diferentes características físicas, dependiendo de la cantidad y la fuente de almidón empleada. Las harinas compuestas con un grado de sustitución de $10 \%$ presentan características físicas parecidas a la muestra de harina de trigo, siendo la sustitución con harina de papa la más cercana. La harina compuesta con quinoa muestra los menores valores de asentamiento y de estabilidad de la cocción, lo cual, indica una disminución en el envejecimiento del producto. Se recomienda estudiar más porcentajes de sustitución y realizar pruebas sensoriales y texturales al producto final. Además, se sugiere medir los cambios físicos y sensoriales del pan durante el almacenamiento y correlacionarlos con los resultados del Mixolab.

Agradecimientos: Se reconoce la financiación del proyecto PHPPF-PIC-08-0000162 de la Universidad Técnica de Ambato, Ecuador. El autor Rodríguez-Sandoval agradece la beca de investigación del Instituto Italo Latino Americano (IILA). Conflicto de intereses: El manuscrito fue preparado y revisado con la participación de todos los autores, quienes declaramos que no existe ningún conflicto de intereses que ponga en riesgo la validez de los resultados presentados. 


\section{BIBLIOGRAFÍA}

1. ABUGOCH, L.; CASTRO, E.; TAPIA, C.; AÑON, M.C.; GAJARDO, P.; VILLAROEL, A. 2009. Stability of quinoa flour proteins (Chenopodium quinoa Willd.) during storage. Int. J. Food Sci. Techn. 44:20132020.

2. AHAMED, N.T.; SINGHAL, R.S.; KULKAMI, P.R.; PALB, M. 1996. Physicochemical and functional properties of Chenopodium quinoa starch. Carbohydr. Polym. 31:99-103.

3. ANDERSON, R.A.; CONWAY, V.F.; PFEIFER, V.F.; GRIFFIN, E.L. 1969. Gelatinization of corn grits by roll - and extrusion - cooking. Cereal Sci. Today. $14: 4-12$

4. ANGIOLONI, A.; COLLAR, C. 2009. Significance of structuring/prebiotic blends on bread dough thermomechanical profile. Eur. Food Res. Techn. 229:603610.

5. CERÓN, A.; HURTADO, A.; OSORIO, O.; BUCHELI, M. 2011. Estudio de la formulación de la harina de papa de la variedad parda pastusa (Solanum tuberosum) como sustituto parcial de la harina de trigo en panadería. Rev. Biotecn. Sect. Agrop. Agroind. 9(1):115-121.

6. CHAUHAN, G.S.; ZILLMAN, R.R.; ESKIN, N.A.M. 1992. Dough mixing and breadmaking properties of quinoa-wheat flour blends. Int. J. Food Sci.Techn. 27:701-705.

7. CODINĂ, G.G. 2008. Influence of flour quality with different extraction ratio on the rheological properties of uniaxial extension induced by the mixolab. J. Agroalim. Proces. Techn.14:119-122.

8. COLLAR, C. 2003. Significance of viscosity profile of pasted and gelled formulated wheat doughs on bread staling. Eur. Food Res. Techn. 216:505-513.

9. COLLAR, C.; BOLLAíN, C.; ROSELL, C.M. 2007. Rheological behaviour of formulated bread doughs during mixing and heating. Food Sci. Techn. Int. 13(2):99-107.

10. GREENE, J.L.; BOVELL-BENJAMIN, A.C. 2004. Macroscopic and sensory evaluation of bread supplemented with sweet-potato flour. J. Food Sci. 69(4):SNQ167-SNQ17.

11. HATHORN, C.S.; BISWAS, M.A.; GICHUHI, P.N.;
BOVELL-BENJAMINA, A.C. 2008. Comparison of chemical, physical, micro-structural, and microbial properties of breads supplemented with sweetpotato flour and high-gluten dough enhancers. LWT Food Sci. Techn. 41:803-815.

12. HENAO-OSORIO, S.; ARISTIZÁBAL-GALVIS, J. 2009. Influencia de la variedad de yuca y nivel de sustitución de harinas compuestas sobre el comportamiento reológico en panificación. Rev. Ing. \& Invest. 29(1):39-46.

13. HOOVER, R. 2001. Composition, molecular structure, and physicochemical properties of tuber and root starches: a review. Carbohydr. Polym.. 45:253-567.

14. KAACK, K.; PEDERSEN, L.; LAERKE, H.N.; MEYER, A. 2006. New potato fibre for improvement of texture and colour of wheat bread. Eur. Food Res. Technol. 224:199-207.

15. KAUR, L.; SINGH, J.; MCCARTHY, O.J.; SINGH, H. 2007. Physico-chemical, rheological and structural properties of fractionated potato starches. J. Food Engin. 82:383-394.

16. LORENZ, K.; COULTER, L. 1991. Quinoa flour in baked products. Plant Foods for Human Nutr. 41:213-223.

17. MISRA, A.; KULSHRESTHA, K. 2003. Potato flour incorporation in biscuit manufacture. Plant Foods for Human Nutr. 58:1-9.

18. MORITA, N.; HIRATA, C.; PARK, S.H.; MITSUNAGA, T. 2001. Quinoa flour as a new foodstuff for improving dough and bread. J. Appl. Glycosci. 48(3):263-270.

19. NAVARRE, D.A.; PILLAI, S.C.; SHAKYA, R.; HOLDEN, M.J. 2011. HPLC profiling of phenolics in diverse potato genotypes. Food Chem. 127(1):34-41.

20. NORMA TÉCNICA ECUATORIANA (NTE) INEN 530. 1980. Harina de trigo. Ensayo de panificación. 2da. Revisión. 1ra ed. Instituto Ecuatoriano de Normalización. Quito. Ecuador. 10p.

21. PARK, S.H.; MORITA, N. 2005. Dough and breadmaking properties of wheat flour substituted by $10 \%$ with germinated quinoa flour. Food Sci. Techn. Int. 11(6):471-476.

22. PARK, S.H.; MAEDA, T.; MORITA, N. 2005. Effect of whole quinoa flours and lipase on the chemical, rheological and breadmaking characteristics of wheat flour. J. Appl. Glycosci. 52(4):337-343. 
23. ROJAS, J.A.; ROSELL, C.M.; BENEDITO DE BARDER, C. 1999. Pasting properties of different wheat flourhydrocolloid systems. Food Hydrocoll. 13:27-33.

24. ROSELL, C.M.; COLLAR, C.; HAROS, M. 2007. Assessment of hydrocolloid effects on the thermomechanical properties of wheat using the Mixolab. Food Hydrocoll. 21:452-462.

25. ROSELL, C.M.; CORTEZ, G.; REPO-CARRASCO, R. 2009. Breadmaking use of andean crops quinoa, kañiwa, kiwicha, and tarwi. Cereal Chem. 84(4):386392.

26. RUALES, J.; NAIR, B.M. 1994. Properties of starch and dietary fibre in raw and processed quinoa (Chenopodium quinoa, Willd) seeds. Plant Foods for Human Nutr. 45:223-246.

27. SANDOVAL, G. 2007. Elaboración de harina precocida de papa (Solanum tuberosum) en autoclave, con las variedades Superchola y Gabriela, para consumo humano. Proyecto CENI. Facultad de Ciencia e Ingeniería de Alimentos. U. Técnica de Ambato. Disponible desde internet en: http://fcial.uta.edu.ec/ proyectos.htm (con acceso 08/03/10).
28. SCANLON, M.G.; ZGHAL, M.C. 2001. Bread properties and crumb structure. Food Res. Int. 34:841-864.

29. SINGH, N.; SINGH, J.; KAUR, L.; SODHI, N.S.; GILL, B.S. 2003. Morphological, thermal and rheological properties of starches from different botanical sources. Food Chem. 81:219-231.

30. SUN, H.; YAN, S.; JIANG, W.; LI, G.; MACRITCHIE, F. 2010. Contribution of lipid to physicochemical properties and Mantou-making quality of wheat flour. Food Chem. 121:332-337.

31. YADAV, A.R.; GUHA, M.; THARANATHAN, R.N.; RAMTEKE, R.S. 2006. Influence of drying conditions on functional properties of potato flour. Eur. Food Res. Techn. 223:553-560.

32. ZAIDUL, I.S.M.; NORULAINI, N.A.N.; OMAR, A.K.M.; YAMAUCHI, H.; NODA, T. 2007. RVA analysis of mixtures of wheat flour and potato, sweet potato, yam, and cassava starches. Carbohydr. Polym. 69:784-791.

Recibido: Agosto 15 de 2011

Aceptado: Marzo 4 de 2012 